\title{
COMMENTARY
}

\section{Therapeutic Role of Sphingosine-1-Phosphate Receptor 2 in the Progression of Esophageal Adenocarcinoma}

\author{
Lindsey Kennedy ${ }^{\star \dagger}$ and Gianfranco Alpini ${ }^{\star \dagger \dagger}$
}

\begin{abstract}
From the Research Service, * Central Texas Veterans Health Care System, Temple; the Department of Medical Physiology, ${ }^{\dagger}$ Texas A\&M Health Science Center, College of Medicine, Temple; and Baylor Scott \& White Health Digestive Disease Research Center, Baylor Scott \& White Health, ${ }^{\ddagger}$ Temple, Texas
\end{abstract}

\begin{abstract}
Esophageal adenocarcinoma (EAC) is the most prevalent form of esophageal cancer in the United States, and its incidence has increased sixfold during the past decade. ${ }^{1,2}$ Specifically, the overall 5-year survival rate of esophageal cancers is only approximately $17 \%$, with an overall survival median time of less than 1 year because of late diagnosis, chemoresistance, and high incidence of metastasis. ${ }^{3}$ Therefore, identification of EAC pathogenesis and potential therapeutic targets is critical. High-grade dysplasia Barrett esophagus (BE) is a precursor to aggressive EAC. Persistent and severe gastroesophageal reflux disease (GERD) has been established as a primary risk factor for the development of both $\mathrm{BE}$ and EAC. ${ }^{4}$ As major components of GERD refluxate, gastric acids and bile acids have drawn great attention for their role in the development of EAC. Gastric acid induces excessive epithelial cell inflammation, which contributes to tumorigenesis-related mutations in TP53 and CDKN2A, both common in advanced BE and EAC. ${ }^{2}$ However, the effects of bile acids on EAC progression are poorly understood.
\end{abstract}

\section{Conjugated Bile Acids Promote EAC Progression via S1PR2 Signaling}

In this issue of the American Journal of Pathology, Liu et $\mathrm{al}^{5}$ report that the conjugated bile acid taurocholic acid (TCA) promotes human EAC invasive growth and cancer stem cell (CSC) expansion through activation of the sphingosine-1-phosphate receptor 2 (S1PR2) pathway and downstream yes-associated protein (YAP) activation. ${ }^{5}$ Previous studies have found that unconjugated bile acids, including deoxycholic acid and chenodeoxycholic acid, induce cyclooxygenase (COX)-2 expression and chronic inflammation, which promote $\mathrm{BE}$ cell proliferation and EAC progression through prostaglandin E2 and inflammationinduced signaling pathways both in vitro and in vivo., Oxidative stress and DNA damage, induced by deoxycholic acid and chenodeoxycholic acid, are also involved in EAC carcinogenesis. ${ }^{6}$ However, several early clinical studies demonstrated that conjugated bile acids, especially TCA and glycocholic acid, but not unconjugated bile acids, are the predominant bile acid signalers in GERD refluxate. ${ }^{8,9}$ This finding is expected because $>99 \%$ of bile acids secreted in bile are conjugated. Conjugated bile acid levels are significantly increased in the refluxate from patients with advanced BE or EAC when compared with patients with benign $\mathrm{BE} .{ }^{10} \mathrm{~A}$ recent study in a rat esophagojejunostomyinduced reflux BE/EAC model showed a significantly higher portion of TCA in refluxate, which was strongly associated with increased incidence of advanced BE and EAC. ${ }^{11}$ Although these results highlighted a critical role for conjugated bile acids in the pathogenesis of EAC, they did

Supported by the Central Texas Veterans Health Care System, the Dr. Nicholas C. Hightower Centennial Chair of Gastroenterology from Baylor Scott \& White Heath (G.A.), and a Veterans Affairs Research Career Scientist Award (G.A.).

Accepted for publication July 10, 2018.

Disclosures: None declared.

The content is the responsibility of the author(s) alone and does not necessarily reflect the views or policies of the Department of Veterans Affairs or the US government.

Address correspondence to Gianfranco Alpini, Ph.D., Central Texas Veterans Health Care System, Texas A\&M Health Science Center, Olin E. Teague Medical Center, 1901 S. First St., Bldg. 205, 1R60, Temple, TX 76504. E-mail: galpini@tamu.edu 
not illustrate the downstream signaling pathways that modulate BE and EAC progression. The study by Liu et $\mathrm{al}^{5}$ provides novel insights in the cancer-promoting role of conjugated bile acids in EAC progression and identifies the role of S1PR2-mediated YAP activation in EAC.

Two human EAC cell lines, OE-19 and OE-33, were used for this study. OE-33, which expresses higher levels of S1PR2 compared with OE-19 cells, has higher motility and invasiveness and is much more sensitive to conjugated bile acid stimulation. Although TCA, glycocholic acid, and S1P promote proliferation of OE-19 and OE-33 cells, TCA and S1P promote the migration, invasion, and growth of OE-33 cells but not OE-19 cells. Interestingly, overexpression of S1PR2 sensitized OE-19 cells to TCA and S1P stimulation, whereas pharmacologic inhibition or genetic knockdown of S1PR2 ameliorated TCA-induced damage. Overexpression of S1PR2 in OE-19 cells increased the invasive capacity of these cells; however, knockdown of S1PR2 in OE-33 cells suppressed invasiveness. These results indicate that S1PR2 is a critical regulator of EAC cell growth independent of conjugated bile acid stimulation, possibly via S1P secretion.

\section{S1PR2 Activation Promotes EAC Invasion and CSC Growth}

$\mathrm{S} 1 \mathrm{P}$ is a well-established signaling molecule that modulates numerous biological processes, including carcinogenesis and cancer development. ${ }^{12}$ The role of S1PR1 and S1PR3 signaling on cancer proliferation, survival, and metastasis has been extensively studied and characterized; however, the role of S1PR2 is controversial. Several studies found that S1PR2 signaling suppresses cancer by inhibition of migration, angiogenesis, and immune cell infiltration. ${ }^{13}$ However, recent reports suggest that S1PR2 activation promotes proliferation, migration, and metastasis through modulation of tumor suppressor genes and formation of filopodia and lamellipodia in cancer cells. ${ }^{13}$ Previously, Liu et $\mathrm{al}^{12}$ and Nagahashi et al ${ }^{14}$ demonstrated that conjugated bile acid activation of S1PR2 promotes invasive growth of cholangiocarcinoma via ERK1/2 activation, COX-2 induction, and inflammation. In addition, S1P promotes transforming growth factor- $\beta$ signaling and migration and invasion in EAC cells; however, pharmacologic inhibition of S1PR2 by JTE-013 treatment reduces these effects. ${ }^{15}$ These observations suggest a potential tumor-promoting role of S1PR2 in EAC, laying the background for the discussed work. Within S1P signaling, sphingosine kinase 1 (SphK1) and SphK2 synthesize S1P from sphingosine. Both SphK1 and SphK2 promote tumorigenesis, especially in gastrointestinal tract cancers. ${ }^{16}$ SphK1 expression correlates with chemoresistance and poorer prognosis of gastroesophageal cancer. ${ }^{17}$ Recent work found that SphK1 is significantly up-regulated in highly invasive esophageal cancer cells and is significantly correlated with invasive capacity. ${ }^{18}$ More studies that focus on the role of SphK1 and SphK2 on EAC progression are required.

The current study found that OE-33 cells (high S1PR2 expression) are poorly differentiated and exhibit mesenchymal cell-like morphologic features, decreased E-cadherin (epithelial marker) expression, and increased motility and invasiveness when compared with OE-19 cells. Furthermore, TCA and S1P enhanced transforming growth factor- $\beta$-induced epithelial-to-mesenchymal transition and promoted CSC expansion in OE-33 cells. These changes were minimal in OE-19 cells (low S1PR2 expression). In addition, OE-33-derived CSC mainly expressed CD44, a mesenchymal marker, whereas OE-19 cells had high expression of LGR5, an intestinal stem cell marker. These findings suggest that TCA and S1P significantly enhance the expansion of OE-19 and OE-33 cells. This work provides convincing evidence of the role of S1PR2 signaling in the development and maintenance of CSCs. This finding is significant because the CSC population contributes to the high incidence of metastasis, chemoresistance, and recurrence of EAC.

\section{S1PR2 Promotes EAC Progression via YAP and $\beta$-Catenin Activation}

Liu et $\mathrm{al}^{5}$ found that the YAP pathway is significantly activated by TCA and S1P through a S1PR2-dependent signaling mechanism. Hippo-YAP signaling is critical for organ size control during development and is a vital regulator of tumorigenesis. As recently published, YAP is a major regulator of the EAC CSC population, and disruption of YAP signaling in EAC cells leads to loss of CSC properties through inhibition of SOX-9 expression. ${ }^{19}$ The pathways upstream of YAP are missing in the current study, but previous work suggests that S1PR2 triggers YAP activation independent of its upstream kinases. ${ }^{20}$ The current study found that administration of TCA was able to induce $\beta$-catenin activation in OE-33 cells, which is consistent with a previously published study. ${ }^{21}$ However, this study did not evaluate the mechanism by which YAP and $\beta$-catenin activation induces EAC invasion; this should be a future area of research. Further studies are necessary to bridge the gap between activated YAP signaling and CD44 expression, with subsequent CSC expansion, in EAC cells.

Gastric acid signaling exacerbates unconjugated bile acid-induced inflammation and EAC growth via acidification of bile acids. ${ }^{22}$ However, the effect of low $\mathrm{pH}$ on conjugated bile acid-induced EAC invasion was not evaluated in the current study and should be analyzed in the future. The role of COX-2 activation by gastric acids, inflammatory cytokines, and bile acids during EAC development has been characterized, ${ }^{23}$ and previous work by Liu et $\mathrm{al}^{24}$ found that conjugated bile acids induce COX-2 expression through S1PR2-mediated signaling in human cholangiocarcinoma cells. The effect of conjugated bile 


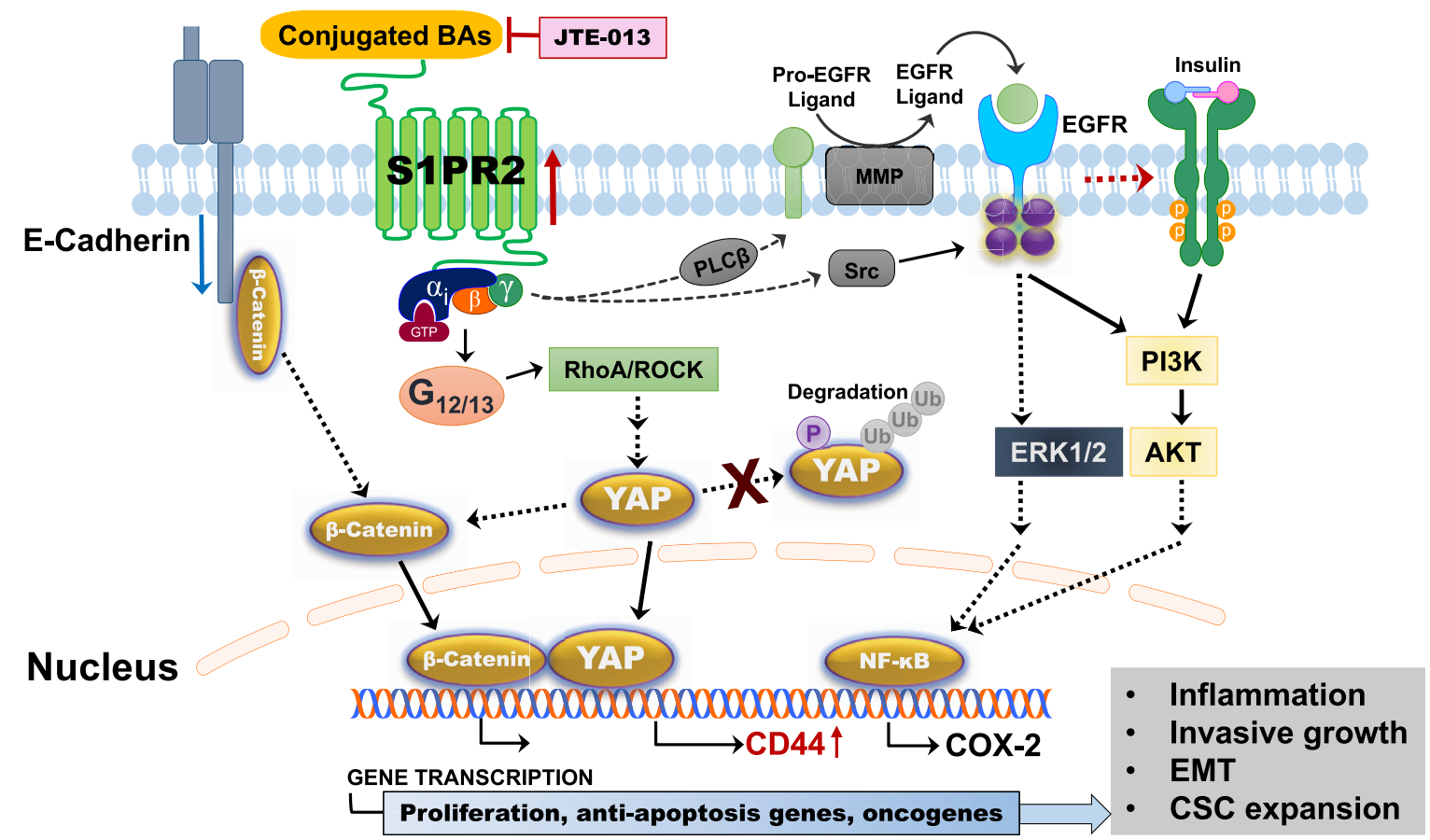

Figure 1 Cell signaling cascade activated by sphingosine1-phosphate receptor 2 (S1PR2) in esophageal adenocarcinoma (EAC). Conjugated bile acids (Bas) activate S1PR2, which then activates the epidermal growth factor receptor (EGFR) via phosphoinositide-specific phospholipase C (PLC)- $\beta$ and Src, followed by ERK1/2 and AKT phosphorylation. Activation of ERK1/2 and AKT signaling induces cyclooxygenase (COX)-2 expression via NF- $K B$. S1PR2 activation promotes the nuclear translocation of yes-associated protein (YAP). In addition, down-regulation of E-cadherin results in disassociation and nuclear translocation of $\beta$-catenin. The nuclear $\beta$-catenin/YAP complex promotes the expression of key genes involved in inflammation, cancer cell proliferation, migration, and cancer stem cell (CSC) expansion. JTE-013 is a chemical antagonist of S1PR2 and may be useful for the treatment of EAC. EMT, epithelial-to-mesenchymal transition; MMP, matrix metalloproteinase; P, phosphorylation; PI3K, phosphatidylinositol 3-kinase; ROCK, Rho-associated protein kinase; Ub, ubiquitination.

acids on COX-2 expression in EAC cells should be investigated in future studies. Understanding the effect of conjugated bile acid signaling on COX-2 expression will help us better understand the signaling mechanisms that regulate inflammation in EAC.

\section{Conclusions and Future Directions}

Although the study by Liu et $\mathrm{al}^{5}$ provides mechanistic insights, it is limited by the lack of in vivo data. Future studies on the role of conjugated bile acids and S1PR2 signaling in animal models is necessary. Human cancer cell xenograft models in null mice are not sufficient for studying S1PR2 in the progression of EAC because they do not recapitulate the inflammatory response noted in EAC progression. Currently, the only model that spontaneously develops EAC and recapitulates GERD is a rat esophagojejunostomyinduced reflux BE/EAC model. ${ }^{11}$ However, gene manipulation in rats is challenging. In addition, a model of advanced BE/EAC that uses human IL-1 $\beta$ transgenic mice in combination with bile acid supplementation in drinking water has been developed and may be useful for future research. ${ }^{6}$ Identifying which animal model will be the best resource for future projects will be a challenge. Another major hurdle is finding an appropriate S1PR2 antagonist because currently there is not a specific S1PR2 antagonist with reasonable bioavailability. For this reason, downstream targeting of S1PR2 signaling, including YAP activation, may be another option. Overall, this study by Liu et $\mathrm{al}^{5}$ provides novel insights into the pathogenesis and development of EAC and identifies the role of conjugated bile acids in EAC invasive growth and CSC expansion. As summarized in Figure 1, conjugated bile acid-induced activation of S1PR2 induces nuclear translocation of YAP and subsequent activation of the epidermal growth factor receptor/ ERK1/2 and phosphatidylinositol 3-kinase/AKT signaling pathways. Previous work from this group has found that activation of ERK1/2 and AKT induces COX-2 expression via NF- $\kappa \mathrm{B} .{ }^{25}$ In addition, down-regulation of E-cadherin results in the nuclear translocation of $\beta$-catenin, and the nuclear $\mathrm{YAP} / \beta$-catenin complex promotes inflammation, cancer cell proliferation, and migration. In conclusion, targeting S1PR2-mediated YAP activation may present a novel therapeutic strategy for the treatment of EAC.

\section{Supplemental Data}

Supplemental material for this article can be found at https://doi.org/10.1016/j.ajpath.2018.07.001. 


\section{References}

1. Siegel RL, Miller KD, Jemal A: Cancer statistics, 2016. CA Cancer J Clin 2016, 66:7-30

2. Dulak AM, Stojanov P, Peng S, Lawrence MS, Fox C, Stewart C, et al: Exome and whole-genome sequencing of esophageal adenocarcinoma identifies recurrent driver events and mutational complexity. Nat Genet 2013, 45:478-486

3. Miller KD, Siegel RL, Lin CC, Mariotto AB, Kramer JL, Rowland JH, Stein KD, Alteri R, Jemal A: Cancer treatment and survivorship statistics, 2016. CA Cancer J Clin 2016, 66:271-289

4. Contino G, Vaughan TL, Whiteman D, Fitzgerald RC: The evolving genomic landscape of Barrett's esophagus and esophageal adenocarcinoma. Gastroenterology 2017, 153:657-673.e1

5. Liu R, Li X, Hylemon PB, Zhou H: Conjugated bile acids promote invasive growth of esophageal adenocarcinoma cells and cancer stem cell expansion via sphingosine 1-phosphate receptor 2 (S1PR2)mediated YAP activation. Am J Pathol 2018, 188:2042-2058

6. Quante M, Bhagat G, Abrams JA, Marache F, Good P, Lee MD, Lee Y, Friedman R, Asfaha S, Dubeykovskaya Z, Mahmood U, Figueiredo JL, Kitajewski J, Shawber C, Lightdale CJ, Rustgi AK, Wang TC: Bile acid and inflammation activate gastric cardia stem cells in a mouse model of Barrett-like metaplasia. Cancer Cell 2012, 21: $36-51$

7. Tselepis C, Morris CD, Wakelin D, Hardy R, Perry I, Luong QT, Harper E, Harrison R, Attwood SE, Jankowski JA: Upregulation of the oncogene c-myc in Barrett's adenocarcinoma: induction of c-myc by acidified bile acid in vitro. Gut 2003, 52:174-180

8. Graffner H, Floren $\mathrm{CH}$, Nilsson A: Conjugated bile salts in gastric aspirates after gastric resection. Scand J Gastroenterol 1984, 19: $116-118$

9. Gotley DC, Morgan AP, Ball D, Owen RW, Cooper MJ: Composition of gastro-oesophageal refluxate. Gut 1991, 32:1093-1099

10. Nehra D, Howell P, Williams CP, Pye JK, Beynon J: Toxic bile acids in gastro-oesophageal reflux disease: influence of gastric acidity. Gut 1999, 44:598-602

11. Chen KH, Mukaisho K, Sugihara H, Araki Y, Yamamoto G, Hattori T: High animal-fat intake changes the bile-acid composition of bile juice and enhances the development of Barrett's esophagus and esophageal adenocarcinoma in a rat duodenal-contents reflux model. Cancer Sci 2007, 98:1683-1688

12. Liu R, Zhao R, Zhou X, Liang X, Campbell DJ, Zhang X, Zhang L, Shi R, Wang G, Pandak WM, Sirica AE, Hylemon PB, Zhou H: Conjugated bile acids promote cholangiocarcinoma cell invasive growth through activation of sphingosine 1-phosphate receptor 2 . Hepatology 2014, 60:908-918

13. Adada M, Canals D, Hannun YA, Obeid LM: Sphingosine-1phosphate receptor 2. FEBS J 2013, 280:6354-6366

14. Nagahashi M, Takabe K, Liu R, Peng K, Wang X, Wang Y, Hait NC, Wang X, Allegood JC, Yamada A, Aoyagi T, Liang J, Pandak WM, Spiegel S, Hylemon PB, Zhou H: Conjugated bile acid-activated S1P receptor 2 is a key regulator of sphingosine kinase 2 and hepatic gene expression. Hepatology 2015, 61:1216-1226

15. Miller AV, Alvarez SE, Spiegel S, Lebman DA: Sphingosine kinases and sphingosine-1-phosphate are critical for transforming growth factor beta-induced extracellular signal-regulated kinase 1 and 2 activation and promotion of migration and invasion of esophageal cancer cells. Mol Cell Biol 2008, 28:4142-4151

16. Pyne NJ, Pyne S: Sphingosine 1-phosphate and cancer. Nat Rev Cancer 2010, 10:489-503

17. Matula K, Collie-Duguid E, Murray G, Parikh K, Grabsch H, Tan P, Lalwani S, Garau R, Ong Y, Bain G, Smith AD, Urquhart G, Bielawski J, Finnegan M, Petty R: Regulation of cellular sphingosine1-phosphate by sphingosine kinase 1 and sphingosine-1-phopshate lyase determines chemotherapy resistance in gastroesophageal cancer. BMC Cancer 2015, 15:762

18. Pan J, Tao YF, Zhou Z, Cao BR, Wu SY, Zhang YL, Hu SY, Zhao WL, Wang J, Lou GL, Li Z, Feng X, Ni J: An novel role of sphingosine kinase-1 (SPHK1) in the invasion and metastasis of esophageal carcinoma. J Transl Med 2011, 9:157

19. Song S, Ajani JA, Honjo S, Maru DM, Chen Q, Scott AW, Heallen TR, Xiao L, Hofstetter WL, Weston B, Lee JH, Wadhwa R, Sudo K, Stroehlein JR, Martin JF, Hung MC, Johnson RL: Hippo coactivator YAP1 upregulates SOX9 and endows esophageal cancer cells with stem-like properties. Cancer Res 2014, 74:4170-4182

20. Yu FX, Zhao B, Panupinthu N, Jewell JL, Lian I, Wang LH, Zhao J, Yuan H, Tumaneng K, Li H, Fu XD, Mills GB, Guan KL: Regulation of the hippo-YAP pathway by G-protein-coupled receptor signaling. Cell 2012, 150:780-791

21. Azzolin L, Panciera T, Soligo S, Enzo E, Bicciato S, Dupont S, Bresolin S, Frasson C, Basso G, Guzzardo V, Fassina A, Cordenonsi M, Piccolo S: YAP/TAZ incorporation in the beta-catenin destruction complex orchestrates the Wnt response. Cell 2014, 158:157-170

22. Zhang F, Altorki NK, Wu YC, Soslow RA, Subbaramaiah K, Dannenberg AJ: Duodenal reflux induces cyclooxygenase-2 in the esophageal mucosa of rats: evidence for involvement of bile acids. Gastroenterology 2001, 121:1391-1399

23. Souza RF, Huo X, Mittal V, Schuler CM, Carmack SW, Zhang HY, Zhang X, Yu C, Hormi-Carver K, Genta RM, Spechler SJ: Gastroesophageal reflux might cause esophagitis through a cytokine-mediated mechanism rather than caustic acid injury. Gastroenterology 2009, 137:1776-1784

24. Liu R, Li X, Qiang X, Luo L, Hylemon PB, Jiang Z, Zhang L, Zhou H: Taurocholate induces cyclooxygenase-2 expression via the Sphingosine 1-phosphate receptor 2 in a human cholangiocarcinoma cell line. J Biol Chem 2015, 290:30988-31002

25. Wang Y, Aoki H, Yang J, Peng K, Liu R, Li X, Qiang X, Sun L, Gurley EC, Lai G, Zhang L, Liang G, Nagahashi M, Takabe K, Pandak WM, Hylemon PB, Zhou H: The role of sphingosine 1phosphate receptor 2 in bile-acid-induced cholangiocyte proliferation and cholestasis-induced liver injury in mice. Hepatology 2017, 65: 2005-2018 